\title{
Educación superior e innovación curricular: resultados de una investigación interinstitucional en red
}

\author{
Higher education and curriculum innovation: results of an \\ institutional network research
}

\author{
Martha Maria Prata-Linhares ${ }^{1}$, Marcos Tarciso Masetto ${ }^{2}$
}

\begin{abstract}
Resumo
En este artículo socializamos la trayectoria de una investigación realizada por un grupo de professores de diferentes universidades brasileñas (FORPEC) que, partiendo de sus indagaciones, construyó una red de investigación e información que compreende diferentes instituciones de enseñanza. Al describir el trayecto del grupo, el texto presenta experiencias innovadoras de currículos de la educación superior que propiciaron significativos cambios pedagógicos y didácticos em sus proyectos. En el transcurso de este proceso fueron estudiados seis proyectos de cursos universitarios con propuestas de innovación. Este análisis permitió elaborar uma conceptualización renovadora para la Educación Superior, identificar nuevas metodologias activas, crear e implantar nuevos proyectos, así como desarrollar investigaciones sobre los procesos de formación de docentes integrantes de estos procesos. La propia trayectoria del grupo puede considerarse un proyecto creador de investigación.

Palabras claves: Innovación educacional. Paradigmas curriculares. Educación superior. Prácticas pedagógicas innovadoras. Investigación en red.
\end{abstract}

\begin{abstract}
In this article we share the paths of an investigation carried out by a group of researchers from different Brazilian universities (FORPEC), which, based on the results of the investigation, built a network of information and research involving various education institutions. While describing this group's pathway, the text presents innovating experiences of curriculums in higher education which provided important and significant pedagogic changes in their projects. During the period of investigation, 6 (six) projects of university courses with innovation proposals were brought up and analyzed. This analysis allowed for the elaboration of innovation concepts for Higher Education, identify active innovating methodologies, create and implant innovative projects in Higher Education, as well as give the start off to investigation concerning the development of the docents integrating the innovating curricular projects. The groups' pathway in itself may be considered an innovative research project.
\end{abstract}

Key Words: Educational innovation. Curricular paradigms. Higher education. Innovative pedagogic practices. Network research.

${ }^{1}$ Doutora em Educação. Docente no Programa de Pós Graduação em Educação da Universidade Federal do Triângulo Mineiro (UFTM), em Minas Gerais.

${ }^{2}$ Doutor em Psicologia da Educação, Livre Docente em Didática. Professor Titular, docente do Programa de Pós Graduação em Educação da Pontifícia Universidade Católica de São Paulo. 


\section{Introducción}

A partir del 2004, el grupo de investigación brasileiro Formación de Profesores y Paradigmas Currículares Innovadores -FORPEC-, investiga currículos innovadores en la Educación Superior existentes tanto en Brasil como en el exterior, discutiendo las concepciones de innovación, los aspectos que identifican tales proyectos como novedosos, así como la formación de profesores que se necesita para la creación y sustentación de esos proyectos.

Este grupo de pesquisa es continuador de un colectivo anterior que investigó el concepto de docencia con profesionalidad en la enseñanza superior, así como las competencias fundamentales para esa docencia y la transformación de las aulas universitarias con los cambios de actitud y las nuevas competencias docentes.

Las investigaciones sobre el desarrollo de innovaciones en el ámbito pedagógico discutieron temas como los siguientes: proceso de aprendizaje de los adultos, formación profesional competente y ciudadana, interacciones entre profesores y alumnos en el proceso de aprendizaje, organización de las disciplinas en el proyecto pedagógico de la carrera, metodologías activas, introducción de las tecnologías de información y comunicación en ambientes virtuales de aprendizaje, proceso de evaluación del aprendizaje, técnicas de evaluación y sus aplicaciones en el cotidiano de la carrera, planeamiento integrado de disciplinas y clases para permitir la consecución de los objetivos de formación esperados.

Otra competencia básica explorada en las investigaciones fue la dimensión política de la docencia en la educación superior, en el sentido de que ella necesita ser contextualizada respecto a los problemas nacionales, con sus debates y con sus consecuencias éticas y ciudadanas, así como relacionadas con las decisiones profesionales y tecnologías incorporadas por los profesores.

El complejo de investigaciones de este grupo estuvo integrado, además, por proyectos de transformación de las clases universitarias de los cursos de graduación (pregrado) en "aulas vivas", dinámicas y de mayor aprovechaiento por parte de los alumnos.

Los resultados de esas investigaciones fueron divulgados em meios científicos e de los resultados mencionados surgieron nuevos problemas de investigación que hoy son examinados por el actual grupo de investigación, el FORPEC.

\section{Nuevo Campo de Investigación}

En efecto, varios grupos de profesores que pasaron a concebir la didáctica de forma innovadora se enfrentaron en sus aulas con una nueva cuestión: las modificaciones pedagógicas y el avance del aprovechamiento de los alumnos está siendo comprometido por diseños curriculares tradicionales que parecen insuperables. Las innovaciones didáticas en las clases demandan transformaciones curriculares.

Partiendo de ese problema, iniciamos una investigación en los cursos de graduación (carreras) que presentaban organizaciones curriculares diferentes a las tradicionales, oportunizando de esa manera las posibilidades para las innovaciones pedagógicas que podrían conducir a una mejora de la formación de los profesionales en cuestión. El camino andado por el FORPEC para el desarrollo de un nuevo campo de investigación fue una construcción por etapas.

\section{Selección de Carreras con Diseños Curriculares Innovadores}

En la primera etapa, la preocupación fundamental fue seleccionar cursos de graduación (carreras) que presentasen organizaciones curriculares diferentes de las tradicionales. Siendo así, se buscó que los cursos seleccionados reunieran las siguientes características: que declarasen una formación más actualizada de sus profesionales, explicitasen bien sus objetivos educacionales, actuación de los 
docentes en equipo, uso de metodologías activas, sistema de evaluación integrado al proceso de aprendizaje y opción por los contenidos atediendo a su aspecto significativo y actualidad científica.

El inicio se dio por los estudios sobre el Programa de Formación de Médicos en Mc Master, en Hamilton, Canadá, en la década de 1970. El punto inicial de la investigación fue el libro Educacion Médica: nuevos enfoques, metas y métodos publicado por Venturelli en 1997, auspiciado por la Organización Panamericana de la Salud (OMS).

El currículo se destacaba por situar como grandes objetivos de la formación la competencia en investigación, el compromiso con la ética profesional y la sensibilidad para las relaciones con los pacientes. El apredizaje se realizaba através de un proceso orientado de descubrimientos, situando el énfasis en la búsqueda de información por parte de los alumnos y el incentivo al aprendizaje interactivo entre pequeños grupos.

El currículo no se organizaba por medio de disciplinas, sino por grandes temas que integraban los contenidos de forma interdisciplinar, así como habilidades, prácticas y actitudes propias del profesional de la salud al enfrentarse con problemas tratados en cada uno de esos grandes temas. Los grandes temas eran desarrollados en lapsus de tiempo de doce a quince semanas cada uno, por grupos de doce alumnos bajo la dirección de un profesor orientador. De esta manera, se abandonaba la organización curricular tradicional de la red disciplinar, con clases de cincuenta y cien minutos desarrolladas con el método expositivo.

Las estratégias de estudio eran selecionadas privilegiando la participación de los alumnos: debates, observación con discusión, lecturas, investigaciones, actividades prácticas con pacientes, actividades simuladas, discusión de casos después de observarlos por circuito cerrado de televisión.

Los alumnos eran obligados a estudiar el año entero, pues el proceso de evaluación era continuo, ofreciendo feed-back de todas las actividades realizadas. Ese retorno existe para ayudar a los alumnos a aprender, pues puede provenir de él propio (autoevaluación), de la evaluación de sus colegas, del profesor-coordinador y, todavía, de los pacientes con los cuales interaccionan durante el proceso de formación. En este proceso los profesores se desempeñan como mediadores del aprendizaje de los alumnos, con una actitud de cooperación y coresposnabilidad por su formación profesional. Estas transformaciones obedecen a ciertos principios que revolucionaron verdades aceptadas como intocables hasta entonces. Entre ellos tenemos: a colocación del alumno en contacto con la realidad profesional desde el primer año de la universidad; asuperación de los pre-requisitos teóricos para de ahí partir hacia la práctica. Se sabe que teoría y práctica pueden y deben estar juntas, facilitando la construcción del conocimiento; el conocimiento no siempre necesita ser adquirido de forma lógica y secuencial. Muchas veces el orden psicológico permite un aprendizaje más significativo; el conocimiento se construye en red y no necesariamente de forma lineal; la responsabilidad personal ante los estudios y la formación profesional, así como la ética en la relación con los colegas, pacientes, profesores y la sociedad en general, son los ejes transversales y los pilares de la formación.

Un segundo proyecto desarrollado por el grupo abrió nuevas perspectivas. El PBL (Problem Based Learning) o APB (Aprendizaje Basado en Problemas) inicialmente pensado y planeado para ser implementado en carreras de formación médica. Nos fundamentamos en Barrows (1994), Berbel (1998), Vleuten, Verwijneng.m, Wijnen, (1996), Ferreira Filho (2002), Komatsu; Zanolli e Lima (2003), Vasquez (1996) y Venturelli (1997).

El PBL propone el desarrollo de un proceso de autoaprendizaje, en contextos de aprendizaje colaborativa, a partir de problemas que son formulados por los docentes del curso y que permiten el alcance de los objetivos educacionales esperados. Tales problemas indican el modelo de 
organización curricular, no más estructurado en disciplinas, sino por módulos o ciclos, en los cuales las ciencias básicas son estudiadas integradamente con las disciplinas clínicas.

De esta manera, se pretende formar profesionales de la salud con espíritu inqueridor, con hábitos permanentes de aprendizaje, con desarrollo de empatía para con los pacientes, con la capacidad de articular prevención y terapias y con abertura para las demandas sociales y políticas en el área de la salud.

Como dice el propio nombre del modelo, el lugar central lo ocupa un problema, una situación real o simulada de la profesión médica, construida intencionalmente por el equipo de profesores que presenta un fenómeno de la realidad que debe ser explicado, o una situación que debe ser resuelta, en términos de procesos y principios subyacentes, por estudiantes que estudian y trabajan en pequeños grupos.

Estos grupos son acompañados por un tutor, a quien cabeindicar el problema, orientar como trabajar en equipo, explicitar todo el proceso de aprendizaje, planear las estrategias y el proceso de evaluación. El problema se presenta sin informaciones anteriores para su solución: es el punto de partida y conductor del proceso de aprendizaje.

Cabe al estudiante explicar todos los aspectos del problema, formular hipótesis, especificar los objetivos de aprendizaje, identificar fuentes de información, buscarlas, realizar las lecturas necesarias, analizar las soluciones, autoevaluarse y recibir evaluación de su desempeño trabajando de forma individual y formando pequeños grupos.

La orientación del estudio, siempre acompañado por un tutor, se desarrolla con carácter interdisciplinar, utilizando e integrando informaciones de diversas disciplinas, que ahora son aprendidas integradamente.

Un tercer modelo de innovación curricular también llamó la atención del grupo de investigación: los Cursos Cooperativos de la Escuela Politécnica de la Universidad de São Paulo (USP), Brasil. En 1989, con carácter experimental, esa escuela de la Universidad de São Paulo implantó las carreras de Ingeniería de Computación, Ingeniería de Producción e Ingeniería Química con un modelo de educación cooperativa, basado en la integración Universidad-Empresa, através de la unión de un esquema académico de alto nivel con fuerte énfasis en actividades de pasantía ofrecidas a los alumnos por las empresas, ampliando la formación profesional con el desarrollo tecnológico, sin perjuicio de la alta calidad de la enseñanza.

Pacheco (2007), describe los pormenores de estos cursos que son realizados en cooperación con las empresas, con una duración de cinco años, durante los cuales el alumno cumple un total de quince módulos. De ellos, nueve son en la Universidad en tiempo integral, a los cuales se les llama Módulos Académicos, y seis en la Empresa, también en tiempo integral, denominados Módulos Prácticos.

Los seis Módulos Prácticos son agrupados de dos en dos en niveles Básico, Intermedio y Avançado. Cada nivel se propone el logro de diferentes objetivos y para lograrlos realizan diversas tareas de aprendizaje incluyendo contactos profesionales, supervisiones de la Empresa y determinados niveles de responsabilidad.

Estos módulos son intercalados durante el año lectivo que se compone de tres cuatrimestres. Eso quiere decir que durante un año los alumnos pueden frecuentar dos módulos académicos y uno de práctica. Ya en el año siguiente, harán dos módulos prácticos y uno académico y así sucesivamente. ¿Qué representan estos módulos prácticos desde el punto de vista de la innovación curricular y didáctico-pedagógica?

Durante los Módulos Prácticos, los alumnos permanecen en la Empresa en tiempo integral y se tornan profesionales contratados por la entidad. Existe un supervisor en la Escuela que le da seguimiento a distancia, así como un funcionario 
de la Empresa que hace el papel de supervisor del trabajo y que, junto a su homólogo de la Escuela $\mathrm{y}$ con el propio practicante forman un equipo de evaluación del aprendizaje del alumno en la Empresa. Los relatos evaluativos son enviados periódicamente al Coordinador del curso.

Durante este módulo práctico, el alumno continuará estudiando e investigando los asuntos que se refieren a su trabajo para cumplir competentemente sus atividades en la Empresa.

Los alunos al retornar al quatrimestre académico traen consigo para las clases todos los problemas, preguntas, asuntos o temas interesantes acumulados durante las prácticas, lo cual enriquece las discusiones a respecto de las diferentes teorías, incentiva la participación de los alumnos y aumenta considerablemente la motivación.

Este currículo, organizado en la forma de módulos académicos intercalados con los módulos prácticos, propicia mayor interacción entre alumnos y profesores, así como el intercambio de informaciones, conocimientos y experiencias de forma más rica. Lo anterior permite que los alumnos adquieran conocimientos académicos y prácticoprofesionales de manera simultánea.

Este modelo curricular introduce un cambio en la forma en que el alumno debe encarar su profesión de ingeniero, así como permite crear una interacción mayor y más efectiva entre los alumnos y profesores, ahora entre ingenieros y estudiantes de ingeniería.

La práctica, tal como concebida y estructurada, se constituye en un verdadera "espacio de clase", y no sólo da la oportunidad de aplicar algunos conocimientos adquiridos en las aulas. La actitud profesional que el alumno asume en relación con la Empresa le ayuda a darse cuenta que aquel es también un lugar privilegiado para aprender, así como para desarrollar la responsabilidad. Esta actitud profesional, asi como el sentido de la capacitación adquirido y consolidado en los módulos prácticos, son llevados para la sala de clases con evidentes ventajas.
Estas investigaciones fueron ampliadas con otras realizadas con proyectos de universidades brasileñas. Así, la Universidad Federal de São Paulo (UNIFESP) desarrolla proyectos en el área de salud, fundamentados en una propuesta de formación interprofesional, integrando alumnos y profesores de las diferentes especialidades médicas e incluyendo profesores de áreas afines.

En entrevistas realizadas con el Profesor Coordinador de la Carrera y con docentes de la misma, acopiamos las informaciones necesarias para entender que el currículo se planifica de forma tal que todos los profesionales de las diferentes especialidades de la salud puedan aprender, en equipos interprofesionales, los conocimientos teóricos y las habilidades prácticas, integrando las disciplinas básicas con las clínicas. En el decurso del currículo los alumos pasan a dedicarse a los estudios y prácticas más específicas de su formación, ya ahora con otra visión de su vida y actividad profesional. El objetivo es que tales profesionales puedan formarse habiendo aprendido a trabajar en equipo con los demás profesionales de diferentes especialidades de la salud.

Todavía en el área de la salud, la Universidad Anhembi Morumbi, de São Paulo, Brasil, creo las Escuelas de Ciencias de la Salud con preocupaciones semejantes: integración entre las carreras y foco en la salud, en el bien estar y en la calidad de vida. En este proyecto estuvimos inmersos durante seis meses, dando seguimiento al desarrollo del proyecto y a la formación de los profesores para construirlo e implantarlo.

Con énfasis en cuidados preventivos, bien estar y calidad de vida, esta propuesta innovadora capacita a los profesionales para la atención de las personas de forma plena: estar bien, sentirse bien y parecer bien.

Este proyecto, con un currículo integrado, flexible y preocupado con innovaciones en la parte didáctica, desarrolla la formación de los profesionales de la salud interconectando las varias especialidades 
del área de la salud, desde la medicina hasta la estética, pasando por la fisioterapia y medicinas alternativas, currículo integrado e interdisciplinar, proceso de aprendizaje por inmersión, énfasis en el aprendizaje en situaciones simuladas con recursos tecnológicos que ofrecen apoyo a estas simulaciones; uso adecuado de las tecnologías de información y comunicación para la investigación en las bibliotecas y bases de datos. También para el ejercicio de prácticas profesionales, formación en contacto con la realidad a través de proyectos interdisciplinares comunitarios, integración efectiva entre teoría y práctica profesional durante toda la carrera, formación de profesores para una docencia que torne las clases más dinámicas y participativas, así como una formación con investigación, con conocimientos interdisciplinares y prácticas consecuentes.

Del mismo modo, estudiamos la Universidad Federal de Paraná - Litoral, Brasil, que presenta hace diez años un proyecto de Universidad, con proyectos de carreras, dentro de un paradigma curricular basado en proyectos, educación y desarrollo sustentable integrado con la acción comunitaria y con la realidad, desarrollo integral del ser humano (KELLER-FRANCO, 2012).

Considerando los proyectos arriba citados como ejemplos del tipo de investigación que el FORPEC viene realizando, en la busca de recursos innovadores de sus currículos y en sus prácticas pedagógicas, la segunda etapa de esta caminata fue la profundización en el concepto de innovación educacional en la enseñanza superior.

\section{Sobre el Concepto de Innovación Educacional}

En efecto, el análisis crítico de estos proyectos que se presentaban muy diferentes de los tradicionales, con excelentes resultados de apredizaje por parte de los alumnos, hizo que nos preocupáramos, a partir de los elementos diferenciadores, por construir una concepción de innovación en la enseñanza superior.
Delineamos la concepción de innovación en la enseñanza superior entendiéndola como un concepto multidimensional, como conjunto de alteraciones que afectan puntos clave y ejes constitutivos de la organización de la enseñanza universitaria, provocadas por cambios en la sociedad o por reflexiones sobre concepciones intrínsecas a la misión de la Educación Superior, y que se vuelve para los aspectos importantes y fundamentales de una nueva propuesta educacional, afectando a toda la Universidad (MASETTO, 2004).

Como innovadores defendemos los cambios en la enseñanza superior que buscan traducir en la vida de las Instituciones las reflexiones actuales sobre concepciones intrínsecas a ese nivel de enseñanza y sua misión en nuestros tiempos.

LaDeclaración Mundial sobre Educación Superior en el Siglo XXI: visión y acción, de la Unesco (1988), por ejemplo, se presenta como un gran marco en la reflexión sobre la misión de la Educación Superior, la cual debe estar vuelta para la formación de personas altamente calificadas y de ciudadanos responsables que practiquen el aprendizaje permanente, así como la promoción, generación y difusión de la investigación y la contribución a la protección y consolidación de los valores de la sociedad. O documento destaca su función ética, así como la recesidad de que la Educación Superior refuerce la cooperación con el mundo del trabajo y que participe del análisis y prevención de las necesidades de la sociedad.

Esta Declaración llega a algunos puntos bastantes concretos que merecen cambios inmediatos como: currículos, métodos pedagógicos de trabajo y aprendizaje, formación continua de profesores, inclusive en el aspecto pedagógico, enfrentamiento a la tecnología y la educación a distancia, comprensión y explotación de los ambientes virtuales.

Autores como Zabalza (2002, 2006), Bain (2007), Cunha $(2006,2010)$ y Masetto (2003, 2007, 2010, 2012) apuntan otros aspectos como indicadores de innovación en la Educación Superior. Entre ellos tenemos: 
a) El proceso de enseñanza repensado para dar énfasis al desarrollo del proceso de aprendizaje de los alumnos en la construcción del conocimiento, en el desarrollo de habilidades e competencias personales y profesionales y en la formación de valores éticos, sociales y políticos presentes en las discusiones de los problemas técnicos.

b) Enseñanza con investigación en la graduación, enfatizando el proceso de investigación.

c) Enseñanza por proyectos.

d) Valoración de la colaboración y de la coparticipación entre profesores y alumnos y entre los propios alumnos en la dinamización del proceso de aprendizaje.

e) Repensar el proyecto pedagógico contando con la participación de aquellos que van a implantar el proyecto pedagógico desde su inicio.

f) Interacción entre profesores, alumnos y dirección como soporte de la construcción de la innovación.

g) Reflexión curricular que permita repensar disciplinas, contenidos, metodologías, evaluación, tiempos y espacios de aprendizaje.

h) Las nuevas organizaciones curriculares se estructuran a partir de experiencias problematizadoras e integradoras del conocimiento producido, contemplando el diálogo entre distintas disciplinas del dominio del saber.

i) Empleo de metodologías activas y participativas; uso de nuevas tecnologías de información y comunicación.

j) Revisión del concepto de proceso de evaluación y de sus prácticas.

k) Transformación del papel y de las actividades del profesor para el de mediador pedagógico entre los alumnos y sus aprendizajes.

1) Docentes con tal visión y actitudes pedagógicas exigen preparación para participar de las innovacions y asumir compromisos con ellas. m) Por fin, toda innovación exige un proceso de seguimiento, a través de un sistema de evaluación adecuado y permanente.

Los tópicos indicados arriba dicen que consideramos como innovaciones en la Educación Superior aquellas alteraciones y transformaciones que se realizan de manera integrada y simultánea para la implantación de un proyecto pedagógico en su totalidad. En nuestro entender, no pueden ser consideradas como innovaciones las alteraciones que se presentan como dispersas, esporádicas, oportunistas, casuales.

Esta consolidación de una concepción de innovación abrió las perspectivas del FORPEC para otros vuelos y osadías. Así, pasamos a desarrollar investigaciones para proyectar e implantar proyectos innovadores. Ellos son: Proyecto de Formación de Profesores para el Curso de Turismo, através del Curso de Posgraduación lato sensu (GAETA, 2012) que se realiza desde hace nueve años en el Centro Universitario del Serviço Nacional de Aprendizagem Comercial (SENAC), São Paulo, Brasil. Proyecto de Formación Pedagógica para Profesores Universitarios con un currículo que integra contribuciones del Arte (PRATA - LINHARES, 2012), en la Universidad de Uberaba, Minas Gerais, Brasil. Thiago Shultze (2012), investigó las contribuciones de un currículo innovador en Turismo, a partir de la ética y de la estética; KellerFranco (2012) de la Universidad Adventista de São Paulo, investigó el currículo por proyectos como un paradigma curricular innovador. Y, como em los proyectos de innovación necessitamos de formas de acompañamiento de los aprendizajes, ZukowskyTavares (2012) de la Universidad Adventista de São Paulo, investigó cómo la presencia de un sistema diferenciado de evaluación se hace necesario para una práctica pedagógica competente en ambientes universitarios. 


\section{Algunas Cuestiones Emergentes}

El momento actual del grupo, en su tercera etapa, apunta para otros desafíos. En la investigación sobre proyectos innovadores percibimos con cierta claridad la importancia fundamental de la participación y del papel de los docentes en la idealización, en la implantación y en la sustentación de un proyecto innovador.

Descubrimos también que algunos proyectos que se iniciaron como innovadores y que no consiguieron mantenerse como tales enfrentaron serios problemas con la permanencia y compromiso de los profesores. De aquí partieron las siguientes preguntas: ¿qué atención les ha sido dada a los profesores que integraron y participaron de un proyecto innovador?, ¿cómo ha sido la selección de ellos?, ¿qué modalidad de formación se desarrolló con relación a ellos para que comprendieran el proyecto innovador?, ¿asumieron un compromiso con este proyecto y se sintieron con competencia para realizarlo?, ¿cómo ha sido el seguimiento dado a los docentes durante la implantación del proyecto innovador?

Buscando respuesta a tales indagaciones, nos encontramos hoy con algunos subproyectos en ejecución sobre la formación de profesores que participan e integran proyectos innovadores. El primero investiga esa formación en un curso innovador de Derecho en la Fundación Getulio Vargas, de São Paulo, Brasil; un segundo proyecto investiga la formación de profesores en la Universidad Federal de Paraná. En esta Universidad, todavía, un terecer proyecto se dedica a estudiar cómo ocurre la formación de profesores en los cursos de licenciatura; un cuarto proyecto se vuelve para la formación de profesores on line y el último investiga la formación de profesores en los proyectos innovadores de la Universidad Federal de São Paulo.

Las conclusiones de nuestras investigaciones hasta el presente, trabajando con proyectos innovadores y los respectivos cambios pedagógicos en las clases que los integran, nos llevan a los siguientes tópicos:

1)Los proyectos innovadores siempre surgen como respuestas a problemas y necesidades actuales de la educación, para las cuales los proyectos educacionales presentes y en curso ya no tienen respuestas adecuadas.

2) Proyectos innovadores sólo se constituyen como tales si las innovaciones suceden en todos los aspectos que constituyen un proyecto educacional y no sólo con transformaciones o alteraciones puntuales.

3)Proyectos educacionales siempre se presentan como un proceso que se va construyendo en tiempos $\mathrm{y}$ espacios concretos, en un contexto histórico preciso de las instituciones y en un movimiento continuo.

4) Proyectos innovadores siempre cuentan con un grupo de educadores que se abre a las nuevas necesidades, asumen un compromiso con la innovación y socializan tales preocupaciones con otros educadores para juntos pensar, planificar y implantar tales proyectos. Sin la participación colectiva de los educadores es imposible llevar a cabo un proyecto innovador. Por eso mismo, en todos los proyectos encontramos integrado a él un proceso de formación continuada de profesores en servicio.

5)Los proyectos innovadores siempre cuentan con la participación efectiva y comprometida de las autoridades administrativas, desde el início mismo del diagnóstico de las necesidades educacionales, así como en el pensar y planificar el nuevo proyecto, en su implantación y evaluación sistemática.

La trayectoria concluida hasta el momento por el FORPEC resultó en la formación de una red de información e investigación, envolviendo diversas universidades. Esa trayectoria también es vista por nosotros como un proyecto innovador de investigación en la educación. 
Este colectivo se constituye como un Grupo Interinstitucional de Investigación, que crea en su organización y en sus colaboraciones relaciones y contactos con otros investigadores que forman un sistema en red.

Así, el grupo está integrado por diez profesores doctores y tres doctorandos, integrantes del cuerpo docente de ocho Instituciones de Educación Superior (IES) de Brasil, pertenecientes a los Estados de São Paulo, Minas Gerais, Bahia y Alagoas.

Estos investigadores delinearon el gran proyecto de investigación (Innovación en la Educación Superior) y lo subdividieron en subproyectos por universidades estudiadas y por temáticas (currículos, prácticas pedagógicas, formación de profesores, formación de profesionales). Estos subproyectos fueron desarrollados por uno o dos de los miembros del grupo, lo cual hicieron intercambiando y discutiendo entre sí y con los membros del grupo informaciones colectadas y los análisis realizados.

Además de que las actividades investigativas se realizaban en forma de red entre todo el grupo, el desarrollo de las investigaciones se llevó a cabo también en colaboración con las universidades brasileñas públicas y privadas en las cuales se encontraban proyectos innovadores ya consolidados o en proceso de implantación.

En esas universidades, los gestores y docentes que construyeron y colocaron en acción los proyectos innovadores se convirtieron en colaboradores del FORPEC para la colecta de datos e informaciones necesarias para la investigación, así como participaron del análisis y discusiones de los resultados obtenidos. En algunas de ellas, se discutieron sugerencias para el avance y desarrollo de los propios proyectos.

Al FORPEC están vinculadas, por medio de las investigaciones y proyectos, la Universidades Federales de Paraná, la de São Paulo, la del Triângulo Mineiro y la de Alagoas. También lo están la Universidad de São Paulo, la Pontificia Universidad Católica de São Paulo, la Universidad
Anhembi-Morumbi de São Paulo, la Universidad Adventista de São Paulo y el Centro Universitario del Servicio Nacional del Comercio de São Paulo.

El FORPEC se constituye como un grupo de investigación en red por sus participantes y por los proyectos de estudio que desenvuelve. Preocupado con la socialización de los resultados de sus investigaciones y con el intercambio de resultados, tiene publicaciones en libros, artículos en revistas y presentaciones de trabajos y estudios en eventos nacionales e internacionales firmados por sus participantes.

Así, este grupo de investigación brasileño cree en la importancia de proyectos curriculares innovadores de cursos de graduación y prácticas pedagógicas para dinamizar y actualizar la Educación Superior en Brasil, así como en la investigación de un proceso de formación continuada para profesores que se comprometen con la creación e implantación de esos proyectos. Cree todavía que estas investigaciones y la socialización de sus resultados podrán contribuir con otras investigaciones que se vienen realizando en América Latina y en Europa y que buscan una nueva era para la Educación Superior.

\section{Referências}

BAIN, K. Lo que hacen los mejores profesores univertsitarios. Valencia: Universitat de Valencia, 2007.

BARROWS, H. S. Como diseñar um currículo de préclínicas basado em problemas. Trujillo: Facultad de Ciências Medicas, Universidad de Trujillo, 1994.

BERBEL, N. A problematização e a aprendizagem baseada em problemas: diferentes termos ou diferentes caminhos? Interface: Comunicação, Saúde, Educação, Botucatu, v. 2, n. 2, p. 139-154, fev. 1998.

VLEUTEN, C. P. M. V. D.; VERWIJNENG, G. M.; WIJNEN, W. H. F. H. Fifteen years of experience with progress testing in a problem-based learning curriculum. Medical Teacher, Basingstoke, v. 18, n. 2, p. 103-109, 1996. 
CUNHA, M. I. (Org.). Trajetórias e lugares de formação da docência universitária: da perspectiva individual ao espaço institucional. Araraquara: Junqueira e Marin, 2010.

.Pedagogia universitária: energias emancipatórias em tempos neoliberais. Araraquara: Junqueira e Marin, 2006.

FERREIRA FILHO, O. F. Visão docente do processo de implementação da aprendizagem baseada em problemas (ABP) no curso médico da UEL. Revista Brasileira de Educação Médica, Rio de Janeiro, v. 26, n. 3, p. 175-183, set-dez, 2002.

GAETA, C. Formação de professores para o ensino superior em cursos de pos-graduação lato sensu: uma opção innovadora no contexto educacional atual. In: MASETTO, M. (Org.). Inovação no ensino superior. São Paulo: Loyola, 2012.

KELLER-FRANCO, E. Inovação na educação superior: o currículo por projeos. In: MASETTO, M. T. (Org.). Inovação no ensino superior. São Paulo: Loyola, 2012.

KOMATSU, R.; ZANOLLI, M.; LIMA, V. Aprendizagem baseada em problemas. Revista Brasileira de Educação Médica, Rio de Janeiro, v. 27, n. 1, 2003.

MASETTO, M. T. Inovação curricular no ensino superior: organização, gestão e formação de professores. In: MASETTO, M. T. (Org.). Inovação no ensino superior. São Paulo: Loyola, 2012. . O professor na hora da verdade. São Paulo: Avercamp, 2010.

MASETTO, M. T. (Org.). Ensino de engenharia: técnicas para otimização das aulas. São Paulo: Avercamp, 2007.

MASETTO, M. T. Inovação na educação superior. Interface: Comunicação, Saúde Educação, Botucatu, v. 8, n. 14, p. 1-18, 2004.

- Competência pedagógica do professor universitário. São Paulo: Summus, 2003.

PACHECO, C. R. F. O estágio e o ensino de engenharia. In: MASETTO, M. T. (Org.). O ensino de engenharia: técnicas para otimização das aulas. São Paulo: Avercamp, 2007.
PRATA-LINHARES, M. M. Contribuições da arte para a formação de professores univeristários. In: MASETTO, M. T. (Org.). Inovação no ensino superior. São Paulo: Loyola, 2012.

UNESCO. Declaracion mundial sobre la educacion superior en el siglo xxi: vision y accion. 1988. Paris: Unesco, 1988.

VASQUEZ, J. Aprendizagen basado em solución de problemas, PBLM. Trujillo: Universidad Nacional de Trujillo, 1996.

VENTURELLI, J. Educacion medica: nuevos enfoques, metas y métodos. Washington: Organización Panamericana de la Salud, 1997.

ZABALZA, M. A. Competencias docentes del profesorado universitario. Calidad y desarrollo profesional. Madrid: Narcea de Ediciones, 2006.

ZABALZA, M. A. La enseñanza universitária, el escenario y sus protagonistas. Madrid: Narcea de Ediciones, 2002.

ZUKOWSKY-TAVARES, C. A avaliação formativa da aprendizagem no ensino superior e o compromisso dos docentes e dos gestores. In: MASETTO, M. T. (Org.). Inovação no ensino superior. São Paulo: Loyola, 2012. 